\title{
CROSS-COUPLED LMS FOR QUADRATIC CONSTRAINED FILTER DESIGN
}

\author{
Miguel A. Lagunas, Ana I. Perez-Neira \\ Modulo D5, Campus Nord UPC \\ Gran Capita s/n \\ 08034 BARCELONA \\ SPAIN \\ e-mail miguelgps.tsc.upc.es
}

\begin{abstract}
Quadratic constrained maximization appears as the central problem in many filter design methods and signal processing applications, whenever the processing or filtering entails the maximization of a signal to noise ratio. Furthermore, self reference systems for MSE filters, beamformers and equalizers which manage, either time or frequency diversity of the desired signal, arise to the same mathematical problem.

This work reports an adaptive algorithm based on two coupled LMS-like algorithms. It is shown how the Lagrange multiplier, involved in constrained minimization problems, plays the role of a gain control in forming the error signal for the filter updates. This gain is power controlled by the output signals keeping the proper equilibrium between the outputs of the two LMS loops.
\end{abstract}

\section{INTRODUCTION}

The formulation of filtering problems use to be in terms of controlling simultaneously two quadratic forms. The first quadratic form is the response of the filter to be designed to the desired signal while the second one is the corresponding response to the noise plus interferers or jammers. The design criteria use to be to minimize the noise contribution, yet preserving the desired signal response constant. The alternative of maximizing the signal contribution is useless since it does not allow dynamic control of the output signal, and it requires an automatic gain control system in addition to the filter stage.

Quadratic constrained maximization is also faced in those systems, like frequency hopping [1] [2], frequency diversity [3] [4] and time diversity spread spectrum for communication systems, that use time slots or frequency bands to transmit replicas of the desired signal. It is easy to prove that filtering out the

This work has been supported by CICYT (TIC960500c10-01,TIC98-0412, TIC98-0703) of Spain and CIRIT (1998SGR-00081) of Catalunya. desired signal from diversity replicas and rejecting interferers or jammers, uncorrelated in the diversity slots or bands, arises to a quadratic constrained maximization problem. The quadratic constrain consists in setting a given value for the cross correlation between the outputs of the filtered version of the diversity components. The same problem appears in the maximization of the spectral self coherence of ciclostationary signals [5] for blind beamforming and filtering. The quadratic constraint in this case is also a cross correlation function.

The solution to the quadratic constrained maximization, in a block processing, reduces to a generalized eigenvalue problem, which involves the two matrixes defining the objective and the constraint. The purpose of this work is to move the inner advantages of the adaptive LMS algorithm to provide sample by sample updates to the solution of those signal processing problems formulated as quadratic constrained maximization. The derivation is based in the gradient of the Lagrangian, with rank one estimates for the involved auto and cross-correlation matrixes. The adequate choice of the step sizes and the crucial role of the Lagrange multiplier are reported. The architecture that supports the resulting algorithm is a cross-coupled LMS loop which resembles the schemes reported some years ago for independent source separation [7].

\section{QUADRATIC CONSTRAINED MAX.}

In order to justify the interest of the adaptive algorithm, to be reported in Section III, this section describes briefly the most interesting applications which are formulated in terms of a quadratic constrained maximization. Quadratic constrained maximization shows up in those problems where the objective is to maximize a signal to noise ratio (SNR), defined in terms of the correlation matrixes for the desired signal $\underline{R}_{s}$ and noise $\underline{\underline{R}} n$ The maximization of the SNR defined in (1), for the filter coefficients denoted with vector $\underline{A}$, 


$$
S N R=\frac{\underline{A}^{H} \cdot \underline{R} \cdot \underline{\underline{R}} \cdot \underline{\underline{A}}}{\underline{A}^{H} \cdot \underline{\underline{R}} \cdot \underline{A}}
$$

can be formulated as an automatic gain control constraint, which sets a constant value for the numerator, and minimizes the denominator.

$$
\begin{aligned}
& \underline{A}^{H} \stackrel{R}{=} \cdot \underline{A}=\phi_{s} \\
& \left.\underline{A}^{H} \cdot \underline{\underline{R}} \cdot \underline{A}\right]_{\min }
\end{aligned}
$$

Note that the same solution applies for the case where the denominator includes the filter output power. Furthermore, for dynamic control reasons in successive stages, located at the output of the filter, it is better to constrain the denominator and maximize the numerator of (3) than the alternative outlined above for the maximization of (1).

$$
\begin{aligned}
& \underline{A}^{H} \cdot\left(\underline{R}_{S}+\underline{\underline{R}}_{n}\right) \underline{A}=\phi_{S} \\
& \left.\underline{A}^{H} \cdot \underline{R}_{S} \cdot \underline{A}\right]_{\max }
\end{aligned}
$$

In these two alternatives, the parameter $\phi_{S}$ plays the role of an automatic gain control that determines the power level of the desired signal at the filter output.

The solution to the quadratic constrained maximization is given by (4)

$$
\begin{aligned}
& \lambda \cdot{ }_{s} \cdot \underline{A}=\underline{R_{n}} \cdot \underline{A} \\
& S N R_{\max }=\lambda_{\text {max }}
\end{aligned}
$$

The same formulation appears in communication systems where, either time, code or frequency, diversity is used for the desired signal [6]. Focussing the case of time diversity, let us assume that the filtering equations are featured by the input snapshots $\underline{X}_{s n}$ and $\underline{X}_{r n}$ and the filter weight vector $A$.

$$
\begin{aligned}
& y_{S}(n)=\underline{A}^{H} \cdot \underline{X}_{s n} \\
& y_{r}(n)=\underline{A}^{H} \cdot \underline{X}_{r n}
\end{aligned}
$$

Indexes $r$ and $s$ indicate that the successive samples forming the snapshots are taken from two different diversity slots. As a consequence, the desired signal is present, as a time replica, in both snapshots and the noise and interferers are uncorrelated from slot $r$ to $s$.

Taking into account that the desired signal is present in the two filter inputs, a suitable criteria to remove undesired signals is to minimize the mean square error (MSE) between the two outputs. In addition, in order to avoid the trivial solution the MSE is constrained by the cross correlation of the outputs. This is formulated in (6).

$$
\begin{aligned}
& \xi=E\left[\left|y_{s}(n)-y_{r}(n)\right|^{2}\right]_{\min } \\
& E\left[y_{s}(n) \cdot y_{r}^{*}(n)+y_{s}^{*}(n) \cdot y_{r}(n)\right]=2 . \phi_{s}
\end{aligned}
$$

Note that the constrained minimization shown in (6) removes those signals which are present either in scenario $r$ or $s$ and those which present in both scenarios present null or small correlation among the diversity slots. In the other hand, the desired signal is enhanced due to its high cross correlation.

Equation (6) is formulated as (7), where $\underline{R}_{s}$ and $\underline{R}_{r}$ are the autocorrelation matrixes for snapshots $\underline{X}_{s n}$ and $\underline{X}_{r n}$, and $\underline{R}_{s r}$ is the crosscorrelation between the mentioned snapshots, i.e. $E\left[\underline{X}_{s n} \cdot \underline{X}_{r n}^{H}\right]$.

$$
\begin{aligned}
& \left.\underline{A}^{H} \cdot \underline{\underline{R}}_{S}+\underline{\underline{R}}_{r}\right]\left.\underline{A}\right|_{\min } \\
& \left.\underline{A}^{H} \cdot \underline{\underline{R}}_{s r}+\underline{\underline{R}}_{r s}\right] \underline{A}=2 \cdot \phi_{s}
\end{aligned}
$$

The frequency dual of the above problem is the case of frequency diversity [4). In this case $r$ and $s$ denote two different frequency bands (the signal forming the snapshots are the complex envelope of the corresponding band-pass signals) .

The single filter design for both diversity components does not guarantee maximum signal to noise ratio. In fact, further improvements can be expected, not only in the average SNR, but also in the segmented SNR when different filters are set for each diversity component as indicated in (8). The use of two different filters is mandatory in maximum likelihood (ML) receivers for communications and ML processing applications.

$$
\begin{aligned}
& y_{s}(n)=\underline{A}_{s}^{H} \cdot \underline{X}_{s n} \\
& y_{r}(n)=\underline{A}_{r}^{H} \cdot \underline{X}_{r n}
\end{aligned}
$$

The constrained minimization is formulated in (9),

$$
\begin{aligned}
& \underline{A}_{S}^{H} \cdot \underline{R}_{s} \cdot \underline{A}_{s}+\left.\underline{A}_{r}^{H} \cdot \underline{\underline{R}}_{r} \cdot \underline{A}_{r}\right|_{\min } \\
& \underline{A}_{s}^{H} \cdot \underline{R}_{s r} \cdot \underline{A}_{r}+\underline{A}_{r}^{H} \cdot \underline{R}_{r s} \cdot \underline{A}_{s}=2 \cdot \phi_{s}
\end{aligned}
$$

and the solution to (9), also found in the literature as the cross-Score solution [5] is shown in (10). 


$$
\begin{aligned}
& \underline{\underline{R}}_{s} \cdot \underline{A}_{s}=\lambda \cdot \underline{\underline{R}} s r \cdot \underline{R}^{-1} \cdot \stackrel{R}{=} r s \cdot \underline{A}_{s} \\
& \underline{R}_{r} \cdot \underline{A}_{r}=\lambda . \stackrel{R}{=}_{r s} \cdot \stackrel{R}{=}_{s}^{-1} \cdot \stackrel{R}{=}_{s r} \cdot \underline{A}_{r}
\end{aligned}
$$

In summary, quadratic constrained maximization appears in many signal processing applications involving signal to noise ratio maximization and diversity techniques, which are central in communications systems which suffer severe fading [6]. Next section reports an adaptive algorithm that updates, at the snapshot rate, the two filters weight vectors using an LMS algorithm.

\section{ADAPTIVE ALGORITHM}

Since the case of two filters is most general and convenient for optimal performance, the adaptive algorithm is described to solve this case. The filtering equations, indicating the dependence with time of both weight vectors, are:

$$
\begin{aligned}
& y_{s}(n)=\underline{A}_{s n}^{H} \cdot \underline{X}_{s n} \\
& y_{r}(n)=\underline{A}_{r n}^{H} \cdot \underline{X}_{r n}
\end{aligned}
$$

After forming the Lagrangian and taking the gradient with respect both weight vectors,

$$
\begin{aligned}
& \Delta_{s}=\underline{R}_{s} \cdot \underline{A}_{s}-\lambda \cdot \underline{\underline{R}} s r \cdot \underline{A}_{r} \\
& \Delta_{r}=\underline{\underline{R}_{r}} \cdot \underline{A}_{r}-\lambda \cdot \underline{\underline{R}} r s \cdot \underline{A}_{s}
\end{aligned}
$$

the adaptive algorithm is formulated as (13), where $\mu_{\mathrm{s}}$ and $\mu_{\mathrm{r}}$ are the corresponding steep sizes.

$$
\begin{aligned}
& \underline{A}_{s, n+1}=\underline{A}_{s n}+\mu_{s} \cdot \underline{\Delta}_{s} \\
& \underline{A}_{r, n+1}=\underline{A}_{r n}+\mu_{r} \underline{\Delta}_{r}
\end{aligned}
$$

To derive an stochastic method the matrixes involved in the deterministic formulation above are estimated by its instantaneous values, i.e. rank one estimation, values. After using the instantaneous estimates and using the filtering equations (11), the stochastic algorithm is formulated as (14)

$$
\begin{aligned}
& \underline{A}_{s, n+1}=\underline{A}_{s n}+\mu_{s n} \cdot \underline{X}_{s n} \cdot\left(\lambda_{n} \cdot y_{r}^{*}(n)-y_{s}^{*}(n)\right) \\
& \underline{A}_{r, n+1}=\underline{A}_{r n}+\mu_{r n} \cdot \underline{X}_{r n} \cdot\left(\lambda_{n} \cdot y_{s}^{*}(n)-y_{r}^{*}(n)\right)
\end{aligned}
$$

It is interesting to note that both the step size, as well as the Lagrange multiplier, are time varying. Also the updating equations reveal in which manner the quadratic constraint modify the error term. This suggest, considering only equation (14.a), that $\mathrm{y}_{\mathrm{r}}(\mathrm{n})$ acts as a time reference for the filter output, and the Lagrange multiplier is just a gain control of the reference. Furthermore, depicting in a scheme the filtering and updating loops, it can be observed in Figure 1 that the architecture is basically two LMS loops which are related by the mentioned gain control.

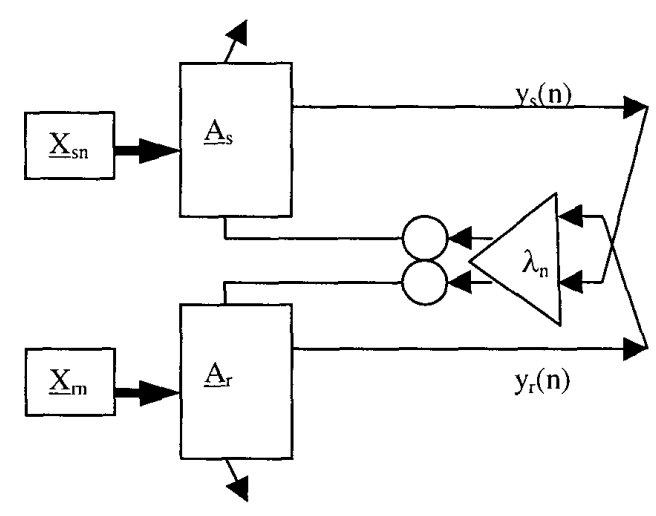

Fig. 1: Cross-coupled adaptive loops to enhance correlated signals

To derive the adequate setting for the gain control $\lambda_{n}$, powers $W_{s n}$ and $W_{m}$ are defined as the instantaneous powers of the corresponding snapshots.

$$
\begin{aligned}
& W_{s n}=\underline{X}_{s n}^{H} \cdot \underline{X}_{s n} \\
& W_{r n}=\underline{X}_{r n}^{H} \cdot \underline{X}_{r n}
\end{aligned}
$$

The step sizes are set in accordance to the rule of the normalized LMS in order to obtain missadjusment errors close to $\alpha$.

$$
\mu_{s n}=2 \alpha / W_{s n} \quad \mu_{r n}=2 \alpha / W_{r n}
$$

In order to obtain the gain control parameter, the updated coefficients from (14) are used in the constraint equation. The proper choice for the parameter is such that the updated coefficients satisfy the constraint. After assuming that the original weights, $\underline{A}_{s n}$ and $\underline{A}_{m}$, already satisfy the constraint, equation (17) is found,

$\lambda_{n}^{2} \cdot \phi_{s} \cdot \alpha+\lambda_{n} \cdot\left(P_{s n}+P_{r n}\right)(1-\alpha)+\phi_{s} \cdot(\alpha-2)=0$

where $\mathrm{P}_{\mathrm{sn}}$ and $\mathrm{P}_{\mathrm{rn}}$ are the instantaneous power of the filter outputs.

$$
P_{s n}=\left|y_{s}(n)\right|^{2} \quad P_{r n}=\left|y_{r}(n)\right|^{2}
$$

As it can be concluded, the value of the gain control depend on the missadjusment parameter. Nevertheless, further insight in the gain parameter can be gained when assuming that, for reasonable levels of missadjusment noise, the parameter $\alpha$ is very small and it can be considered zero in (18). With this approximation the gain parameter can be computed directly from the automatic gain parameter $\phi_{\mathrm{s}}$ and the powers at the filter's outputs.

$$
\lambda_{n}=\frac{\phi_{s}}{P_{s n}+P_{r n}}
$$


This expression reveals that, after convergence, the gain control tends to one, since the automatic gain will be equal to two times the power of the desired signal and the denominator, after adequate filtering, will be the same. Before convergence, the setting of the designer for the automatic gain control dictates different values of the gain control. It should be reminded that the value of the constraint is obtained either from the input power of the desired signal or from the filter weights; in other words, in order to have adequate dynamic range in the coefficients and/or in the filtering operation, when using finite register length both for the coefficients and for the input signals, it is necessary to select the corresponding value for the automatic gain control $\phi_{\mathrm{s}}$.

Note that any realistic implementation of the algorithm requires smoothed version of the instantaneous powers defined before. Values above 0.99 for the smoothing parameter prove to be adequate to preserve the performance expected from the initial setting for missadjusment noise. Finally, it is important to remark that the cross-coupled architecture of Figure 1 is close to those schemes proposed some years ago for independent source separation.

\section{SIMULATIONS}

Using two different scenarios for $r$ and $s$ time slots, the desired signal was present as a replica with SNR equal to $10 \mathrm{~dB}$ in independent white gaussian noise. The signal consists in a digital phase modulated signal containing the same symbols in both scenarios. In addition, two uncorrelated interferers were added to the desired with SNR equal to $10 \mathrm{~dB}$ above the desired. Figure 2 depicts the learning curve of the cross-coupled algorithm (smoothed square error between the two filter outputs) for a missadjusment of $10 \%$. Figure 3 shows the evolution of the gain control parameter.

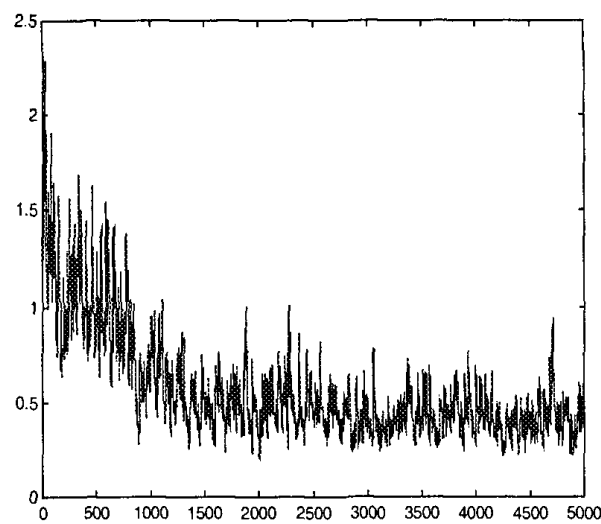

Fig. 2: Learning curve of the algorithm

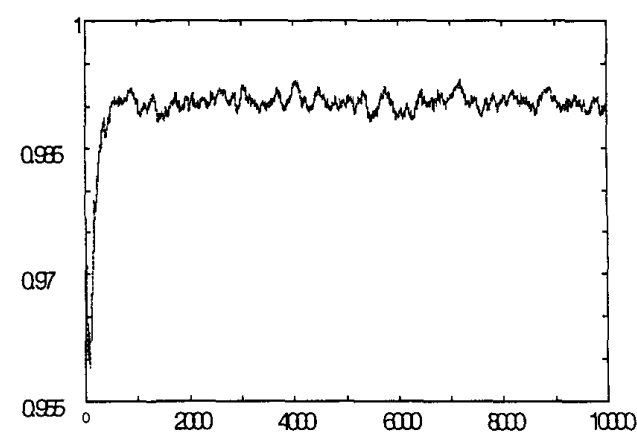

Fig. 3: Evolution of the gain control.

\section{CONCLUSSIONS}

An adaptive algorithm, based in the instantaneous gradient, has been reported for quadratic constrained maximization. The corresponding architecture shows a coupled pair of LMS loops controlled by a gain control for self reference. The gain control mix up the two filters outputs in order to achieve the proper control of both adaptive loops. The gain control is power controlled by the logarithmic difference between the automatic control setting and the corresponding filter outputs. In this way, the architecture exhibits dynamic control of weight and input finite length representations. The algorithm and the architecture are of interest in those problems involving SNR maximization and those problems where the desired signal presents time or frequency diversity.

\section{REFERENCES}

[1] M.Najar, M.A.Lagunas. "Adaptive array beamforming for frequency hopping modulation". Signal Processing VIII, Vol. II, pp. 939-942. Sept 1996. Trieste, Eusipco 96. Italy.

[2] D. Torrieri, K. Bakhru. "Frequency compensation in an adaptive antenna system for frequency-hopping communications". IEEE Trans. on Aerospace Electronics Systems. Vol. AES-23, no. 4, pp. 448-466, July 1987.

[3] G. K. Kaleh. " Frequency diversity spread spectrum communications to counter band-limited gaussian interference". IEEE Trans. on Communications, Vol. 44, No. 7, pp. 886-893, July 1996.

[4] M. A. Laguna, A.I. Perez, M. G. Amin, J. Vidal. " Spatial processing for frequency diversity schemes". Submitted to IEEE- SP.

[5]B.G. Agee, S.V. Schell, W.A. Gardner. "Spectral self coherence restoral: A new approach to blind adaptive signal extraction using antenna arrays". Proc. IEEE, Vol. 78, pp. 753-767, April 1990.

[6] J. Proakis. "Digital communications", Third Edition, Mac Graw-Hill, Chapters 12-15, 1995.

[7] Y. Bar-ness, J. Rokach. "Cross-coupled bootstrapped interference canceller". Proc. Intern. Symp., IEEE-AP, pp. 292-295, 1981. 